\title{
Antimutagenic and Antioxidant Activities of Thai Rice Brans
}

\author{
Orapin Insuan ${ }^{1,2}$, Arpamas Chariyakornkul ${ }^{1}$, Yuwada Rungrote ${ }^{1}$, Rawiwan Wongpoomchai ${ }^{1}$ \\ ${ }^{1}$ Department of Biochemistry, Faculty of Medicine, ${ }^{2}$ Department of Medical Technology, Faculty of Associated Medical Sciences, Chiang Mai \\ University, Chiang Mai, Thailand
}

\begin{abstract}
Background: Rice bran is the outer layer of the rice grain, and contains high amounts of bioactive phytochemicals. Here, we investigated and compared chemopreventive properties of purple and white rice bran extracts.

Methods: Rice bran was extracted with dichloromethane and methanol. Chemical constituents in the extracts were analyzed by colorimetric assay and high performance liquid chromatography. The mutagenicity and antimutagenicity of the extracts were determined via the Salmonella mutation assay. The anticarcinogenic enzyme induction and antioxidant activities of the extracts were examined using Hepa1c1c7 cells and 2,2-diphenyl-1-picrylhydrazyl radical scavenging assay, respectively.

Results: The methanol extracts of rice bran contained high amounts of phenolic acids, flavonoids, anthocyanins, and phytic acid, whereas large amounts of $\gamma$-oryzanol and vitamin $E$ were presented in the dichloromethane extract. None of the extracts were mutagenic to Salmonella typhimurium. All rice bran extracts had strong antimutagenic effects against aflatoxin $\mathrm{B}_{1}$ - and 2-amino-3,4-dimethylimidazo [4,5-f]quinoline-induced mutagenesis. The inhibitory effect against 2-aminofluorene-induced mutagenesis was found in the dichloromethane extract, while only the methanol extract of purple rice bran exhibited antimutagenic effects against benzo(a)pyrene. None of the extracts induced quinone reductase activity in Hepa1c1c7 cells. Additionally, the greatest antioxidant capacity was found in the methanol extract of purple rice bran.

Conclusions: The methanol extract of purple rice bran containing high amount of phenolic acids, flavonoids, anthocyanins, and phytic acid showed the most effective antioxidant and antimutagenic activities by inhibiting mutagenic metabolizing enzymes and/or scavenging free radicals. These results demonstrate the nutritional and medical value of Thai rice for cancer prevention.
\end{abstract}

(J Cancer Prev 2017;22:89-97)

Key Words: Oryza sativa L., Mutagenicity, Antimutagenicity, Antioxidant

\section{INTRODUCTION}

The development of cancers involves the mutation of proto-oncogenes and tumor suppressor genes, leading to uncontrolled cell growth. ${ }^{1}$ While surgery and chemotherapy remain the major treatments for cancer, these treatments may have serious side effects. Research on the cancer chemopreventive effects of phytochemicals that naturally occur in vegetables and fruits aims to provide alternative therapies. ${ }^{2}$ Cancer chemopreventive agents have been reported to interfere with several steps in car- cinogenesis, including protecting DNA from oxidative damage, inhibiting carcinogen activation, and activating carcinogen detoxifying systems. ${ }^{3}$

Rice (Oryza sativa L.) is a staple food source consumed by a majority of the world's population, mainly Asian. ${ }^{4}$ Rice bran, a byproduct of rice milling, contains many bioactive phytochemicals, such as phenolic compounds, tocopherols, tocotrienols, $\gamma$-oryzanol, and phytic acid. ${ }^{5}$ These phytochemicals are able to promote antioxidant and anti-inflammatory activity and suppress tumor progression. ${ }^{6.7}$ Although white rice is predomi-

Received April 9, 2017, Revised May 7, 2017, Accepted May 8, 2017

Correspondence to: Rawiwan Wongpoomchai

Department of Biochemistry, Faculty of Medicine, Chiang Mai University, Su Thep, Mueang Chiang Mai District, Chiang Mai 50200, Thailand Tel: +66-53-94-5225, Fax: +66-53-89-4031, E-mail: rawiwan.wong@cmu.ac.th, ORCID: Rawiwan Wongpoomchai, http://orcid.org/0000-0001-6999-8436

Copyright (C) 2017 Korean Society of Cancer Prevention

(c) This is an Open Access article distributed under the terms of the Creative Commons Attribution Non-Commercial License (http://creativecommons.org/licenses/by-nc/4.0) which permits unrestricted non-commercial use, distribution, and reproduction in any medium, provided the original work is properly cited. 
nantly consumed, pigmented rice is an alternative food known for its health-promoting effects. ${ }^{8}$ Our group found that purple rice bran extract exhibited antigenotoxic effects on aflatoxin $B_{1}$ $\left(\mathrm{AFB}_{1}\right)$-induced micronucleus formation in rat liver by alteration of several xenobiotic metabolizing enzymes in $\mathrm{AFB}_{1}$ metabolism. ${ }^{9}$ A number of varieties of rice and their potential beneficial effects have been investigated. ${ }^{10}$ However, comparative study of the biological activities of pigmented and white glutinous rice bran is still lacking. We thus examined the mutagenic and antimutagenic effects of both hydrophilic and lipophilic extracts in the bran of purple and white rice. The respective antioxidant and anticarcinogenic enzyme inducing activities were also determined.

\section{MATERIALS AND METHODS}

\section{Chemicals}

Standard mutagens, including $\mathrm{AFB}_{1}$, sodium azide $\left(\mathrm{NaN}_{3}\right)$, and 2-aminoflurene (2-AF) were purchased from Sigma-Aldrich (St. Louis, MO, USA). 2-aminoanthracene (2-AA), 2-(2-furyl)-3-(5-nitro-2-furyl)-acrylamide (AF-2), 2-amino-3,4-dimethylimidazo[4,5-f] quinoline (MeIQ), and benzo(a)pyrene (B(a)P) were obtained from Wako Pure Chemicals Industries Ltd. (Osaka, Japan). 2,2-Diphenyl-1-picrylhydrazyl (DPPH) and ascorbic acid were purchased from Merck (Darmstadt, Germany). The phenolic acids, flavonoids, anthocyanins, sodium phytate, $\gamma$-oryzanol, and tocols standards for chemicals analysis were high performance liquid chromatography (HPLC) grade. All other chemicals were analytical grade.

\section{Preparation of rice bran extracts}

Glutinous rice (Oryza sativa L. var. indica) was obtained from Dr. Korawan Sringarm in Faculty of Agriculture, Chiang Mai University (Chiang Mai, Thailand). The bran of purple rice (cv. Kum Doi Saket) and white rice (cv. RD6) was collected using a laboratory milling machine and sieved from the rough rice. The lipophilic constituents of rice bran were extracted by soaking three times in dichloromethane for 48 hours. After filtration, the residues were macerated in methanol three times for 48 hours to obtain the hydrophilic ingredients. The filtrates were evaporated and lyophilized, resulting in dichloromethane and methanol extracts.

\section{Determination of chemical constituents}

The total phenolic compounds were determined according to the Folin-Ciocalteu method using gallic acid as a standard. The total flavonoid content was determined according to the alumi- nium chloride colorimetric method using catechin as a standard. ${ }^{11}$ The total content of phytic acid was determined by the method of Gao et al. ${ }^{12}$ using sodium phytate as a standard. The analyses of phenolic acids, flavonoids, anthocyanins, vitamin E, and $\gamma$-oryzanol contents were performed by HPLC. Phenolic acids in rice bran extracts were determined using a reverse-phase $\mathrm{C} 18$ column $(4.6 \times 250 \mathrm{~mm}, 5 \mu \mathrm{m})$ and analyzed using a gradient mobile phase consisting of 3\% acetic acid in deionized water and methanol at the flow rate of $1 \mathrm{~mL} / \mathrm{min}$. The injection volume was $10 \mu \mathrm{L}$ and phenolic acids were monitored at the wavelengths of $260,280,320$, and $360 \mathrm{~nm} .{ }^{13}$ The content of phenolic acids was calculated using calibration curves of gallic acid, protocatechuic acid, 4-hydroxybenzoic acid, chlorogenic acid, vanillic acid, syringic acid, p-coumaric acid, ferulic acid, and ellagic acid and expressed as milligrams per gram extract.

The HPLC analysis of flavonoids was determined using a C18 column $(4.6 \times 250 \mathrm{~mm}, 5 \mu \mathrm{m})$ and rice bran extracts were eluted with the gradient elution of mobile phase consisting of $1 \%$ acetic acid in deionized water and $1 \%$ acetic acid in methanol. Flavonoids were detected at the wavelength of $280 \mathrm{~nm}$ at the flow rate of $1 \mathrm{~mL} / \mathrm{min} .{ }^{14}$ Quantities of flavonoid contents were measured using calibration curves of standards consisting of catechin, epicatechin, rutin, quercetin, luteolin, and apigenin. For the analysis of anthocyanins, the extracts were analyzed using a gradient elution of $0.1 \%$ trifluoroacetic acid (TFA) in water and $0.1 \%$ TFA in methanol at the wavelength of $520 \mathrm{~nm} .{ }^{15}$ Cyanidin-3-glucoside, cyanidin-3-rutinoside, peonidin-3-glucoside, and malvidin-3-glucoside were used as standards.

The $\gamma$-oryzanol content was performed under isocratic elution with mobile phase of $0.5 \%$ acetic acid in acetonitrile, methanol, and dichloromethane (45:40:15, v/v/v), and quantified using a UV detector at a wavelength of $325 \mathrm{~nm} .{ }^{16}$ The vitamin $E$ was analyzed by a normal phase VertiSep ${ }^{\mathrm{TM}}$ UPS silica column $(250 \times 4.6 \mathrm{~mm}$, $5 \mu \mathrm{m})$. The mobile phase was composed of hexane/ isopropanol/ethyl acetate/acetic acid (97.6:0.8:0.8:0.8, v/v/v/v) with a flow rate of isocratic elution of $1 \mathrm{~mL} / \mathrm{min}$. The $\alpha, \beta, \gamma$, and $\delta$ forms of tocopherols and $\alpha, \beta$, and $\delta$ forms of tocotrienols were analyzed by a fluorescence detector at an excitation wavelength of $294 \mathrm{~nm}$ and emission wavelength of $326 \mathrm{~nm} .^{17}$

\section{Mutagenicity test}

The mutagenic activity of rice bran extracts was performed using Salmonella typhimurium tester strains TA98 and TA100, with and without an exogenous metabolic activation by $\$ 9$ mix. ${ }^{18}$ The bacterial tester strains were kindly supplied by Dr. Kei-ichi Sugiyama in National Institute of Health Science (Tokyo, Japan). 
For the mutagenicity, various concentrations $(0.2-5.0 \mathrm{mg} / \mathrm{plate})$ of rice bran extracts and the bacterial culture were incubated with phosphate buffer or S9 mix. Then, top agar containing $0.05 \mathrm{M}$ L-histidine and 0.05 M D-biotin was added and poured onto a minimal glucose agar plate. After the plates were incubated at $37^{\circ} \mathrm{C}$ for 48 hours, the number of histidine-independent revertant colonies was counted. The 2-AA and AF-2 were used as a positive control in the presence or absence of metabolic activation. Dimethyl sulfoxide (DMSO) was used as a negative control. The extract was identified as a possible mutagen when the revertant colonies produced by an extract were 2 times higher than spontaneous revertant colonies.

\section{Antimutagenicity test}

$\mathrm{AFB}_{1}$ was used as a standard mutagen in strain TA98, whereas 2-AF, MeIQ, and $\mathrm{B}(\mathrm{a}) \mathrm{P}$ were used in strain TA100. Various concentrations (0.04-1.0 mg/plate) of rice bran extracts, standard mutagens, and the tester strains were incubated with $\mathrm{S} 9 \mathrm{mix}$. Then, the mixture was incubated, mixed with top agar, and poured onto a minimal glucose agar plate as previously described. After incubation, the number of histidine-independent revertant colonies was counted and compared with the positive control. Antimutagenicity was expressed as percentage of inhibition of mutagenicity. The antimutagenic activity was indicated when the percentage inhibition was more than $30 .^{18}$

\section{Determination of the induction of quinone reductase activity}

The induction activity of quinone reductase was measured in murine hepatoma cells according to Prochaska et al. ${ }^{19}$ Briefly, 10,000 cells/well of Hepalc1c7 cells (ATCC CRL-2026) were seeded onto 96 -well plates and incubated at $37^{\circ} \mathrm{C}$ and $5 \% \mathrm{CO}_{2}$ for 24 hours. The cells were incubated with the various concentrations ranged from 50 to $200 \mu \mathrm{g} / \mathrm{mL}$ of rice bran extract or $0.4 \%$ DMSO (negative control) for 24 hours. To measure quinone reductase activity, the reaction mixture containing $0.5 \mathrm{M}$ Tris buffer $\mathrm{pH} 7.4,7.5 \mathrm{mM}$ flavin adenine dinucleotide, $150 \mathrm{mM}$ glucose-6-phosphate, $50 \mathrm{mM} \mathrm{NADP}{ }^{+}, 2 \mathrm{U} / \mathrm{mL}$ glucose-6-phosphate dehydrogenase, $50 \mathrm{mM}$ menadione, and MTT dye was added after breaking cells with $0.8 \%$ digitonin. Then, $0.3 \mathrm{mM}$ dicoumarol was added and the NADPH-dependent menadiol-mediated reduction of MTT was measured at $620 \mathrm{~nm}$. The cell density was measured by crystal violet staining. The specific activity of quinone reductase and the induction fold of rice bran extract was calculated.

\section{Determination of antioxidant activity}

The antioxidant activities of rice bran extracts were measured via the DPPH radical scavenging activity assay. ${ }^{20}$ One hundred $\mu \mathrm{L}$ of rice bran extract were added into $200 \mu \mathrm{L}$ of DPPH solution in methanol. The absorbance of reaction mixture was determined at $517 \mathrm{~nm}$ at 0 and 30 minutes. The decreased absorbance indicated an increase of the DPPH radical scavenging activity, which was calculated according the formula as DPPH radical scavenging activity $(\%)=\left[\left(\mathrm{A}_{517 \mathrm{~nm}(\mathrm{t}=0)}-\mathrm{A}_{517 \mathrm{~nm}(\mathrm{t}=30)}\right) / \mathrm{A}_{517 \mathrm{~nm}(\mathrm{t}=0)}\right] \times 100(\mathrm{t}=$ 0 : absorbance of the control at 0 minute and $t=30$ : absorbance of the antioxidant at 30 minutes). The effective concentration of the extract that reduced $50 \%$ of DPPH radical formation (EC50) was calculated using a calibration curve in the linear range by plotting concentration of the extract vs. percentage of radical scavenging activity.

\section{Statistical analysis}

Three separate measurements were tested. The results of chemical constituents and antioxidant activity were expressed as mean $\pm \mathrm{SD}$, while the number of revertant colonies and percent inhibition in Salmonella mutation assay were expressed as mean \pm SEM. Differences between the extracts were determined by one-way ANOVA analysis and $P<0.05$ was considered as significant.

Table 1. Chemical constituents in rice bran extracts

\begin{tabular}{|c|c|c|c|c|c|}
\hline Extract & $\begin{array}{l}\text { Total phenolic content } \\
\text { (mg GAE/g extract) }\end{array}$ & $\begin{array}{l}\text { Total flavonoid content } \\
\text { (mg CE/g extract) }\end{array}$ & $\begin{array}{l}\text { Total phytic content } \\
\text { (mg SP/g extract) }\end{array}$ & $\begin{array}{l}\text { Total vitamin } \mathrm{E} \\
(\mu \mathrm{g} / \mathrm{g} \text { extract })\end{array}$ & $\begin{array}{c}\text { Total } \gamma \text {-oryzanol } \\
\text { (mg/g extract) }\end{array}$ \\
\hline \multicolumn{6}{|l|}{ Purple rice bran } \\
\hline Dichloromethane & $4.88 \pm 0.61$ & $3.55 \pm 0.48$ & $2.58 \pm 0.37$ & $1,207.26 \pm 14.27$ & $20.07 \pm 0.15$ \\
\hline Methanol & $88.51 \pm 6.37$ & $59.08 \pm 2.38$ & $23.11 \pm 0.49$ & $108.67 \pm 3.15$ & $1.44 \pm 0.06$ \\
\hline \multicolumn{6}{|l|}{ White rice bran } \\
\hline Dichloromethane & $4.90 \pm 0.38$ & $1.68 \pm 0.56$ & $2.59 \pm 0.40$ & $1,137.80 \pm 15.28^{\mathrm{a}}$ & $13.99 \pm 0.17^{\mathrm{a}}$ \\
\hline Methanol & $26.39 \pm 4.62^{b}$ & $11.62 \pm 0.78^{\mathrm{b}}$ & $12.09 \pm 0.67^{b}$ & $119.59 \pm 2.01$ & $4.05 \pm 0.10^{\mathrm{b}}$ \\
\hline
\end{tabular}

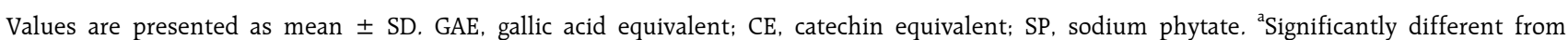
dichloromethane extract of purple rice bran $(P<0.05)$. ' Significantly different from methanol extract of purple rice bran $(P<0.05)$. 


\section{RESULTS}

\section{Chemical constituents in rice bran extracts}

The methanol extracts of rice bran contained higher amounts of total phenolic, flavonoids, and phytic acid contents than the dichloromethane extracts, whereas large amounts of total vitamin $\mathrm{E}$ and $\gamma$-oryzanol were found in the dichloromethane extracts (Table 1). Greater amounts of these phytochemicals were observed in purple rice bran compared to white rice bran. Protocatechuic acid, vanillic acid, and p-coumaric acid were the major phenolic acids in purple rice bran. Only ferulic acid was found in the methanol extract of white rice bran. In addition, anthocyanins were only detected in the methanol extract of purple rice bran and the major anthocyanins were cyanidin-3-glucoside and peonidin-3-glucoside as shown in Figure 1. Due to trace amounts of $\beta$-tocotrienol detected in rice samples, the abundance of seven isomers of vitamin $\mathrm{E}(\alpha-, \beta-, \gamma-$, and $\delta$-tocopherols and $\alpha$-, $\gamma$-, and $\delta$-tocotrienols) in rice bran extracts
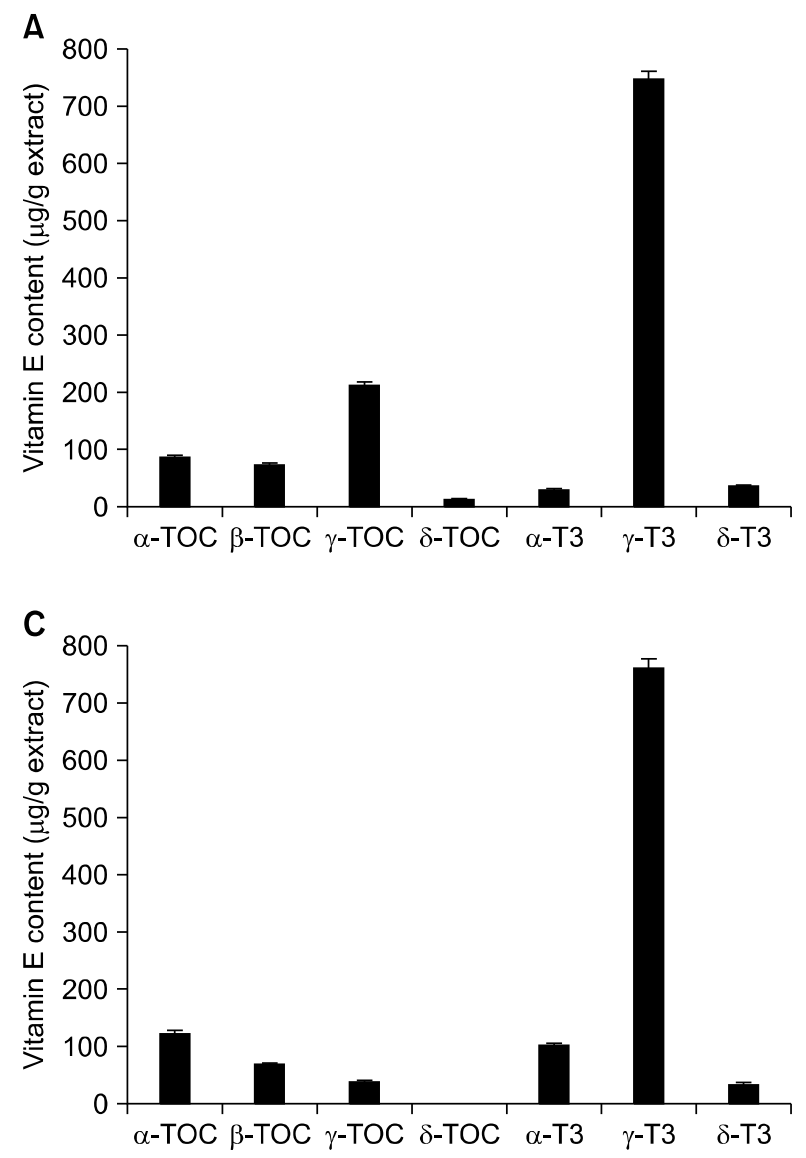

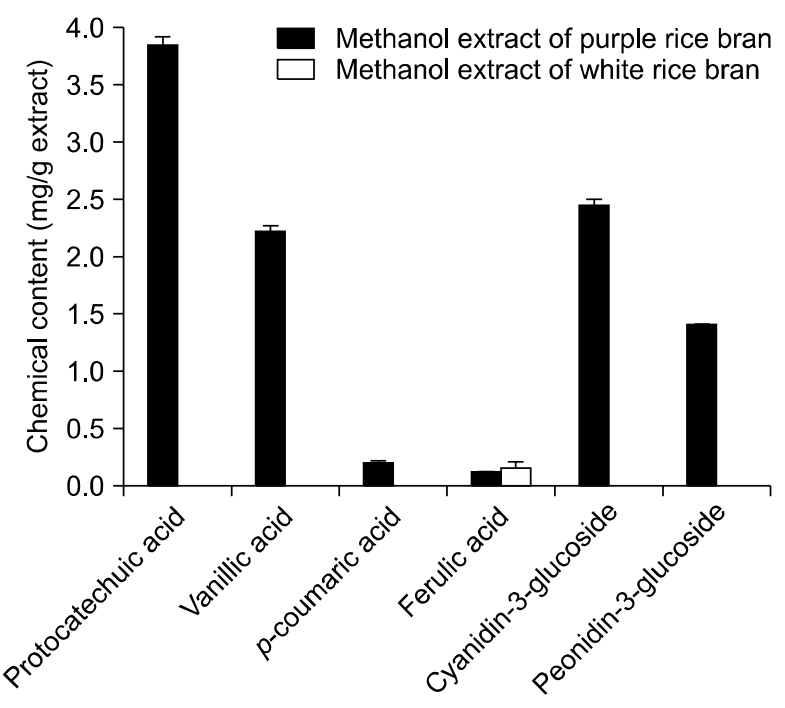

Figure 1. Comparison of some phenolic compounds in the methanol extracts of rice bran.

\section{B}

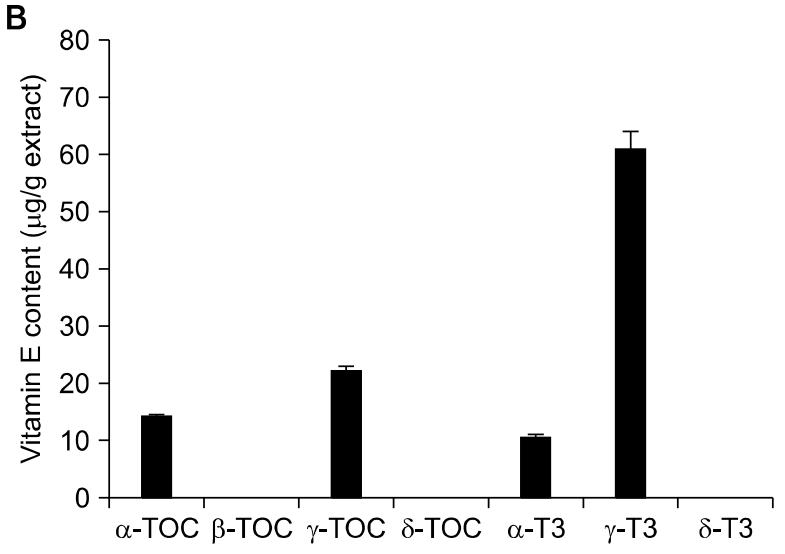

D

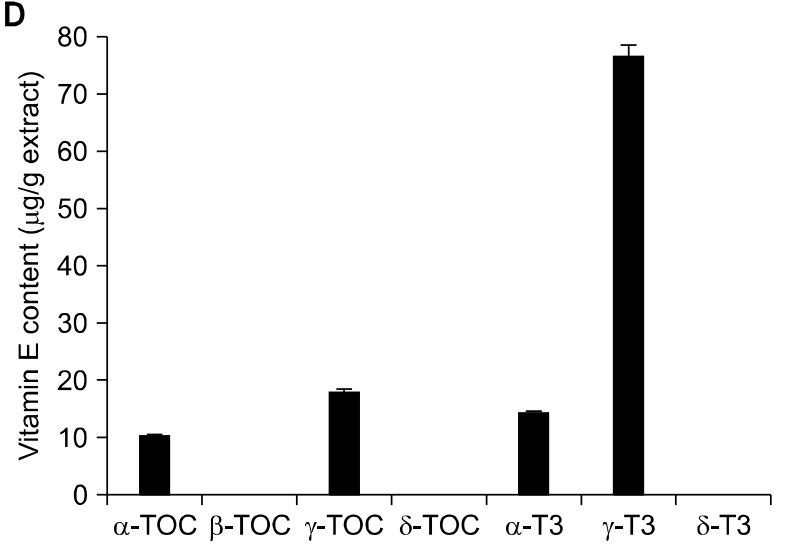

Figure 2. Distribution of tocopherol and tocotrienol contents in rice bran extracts. (A) Dichloromethane extract of purple rice bran. (B) Methanol extract of purple rice bran. (C) Dichloromethane extract of white rice bran. (D) Methanol extract of white rice bran. TOC, tocopherol; T3, tocotrienol. 
was analyzed in the present study. The analysis showed that $\gamma$-tocotrienol was the major form of vitamin $\mathrm{E}$ in rice bran extracts (Fig. 2). The highest amount was found in the dichloromethane extract of white rice bran. $\delta$-tocopherol was only found in the dichloromethane extract of purple rice bran. Some tocols, including $\beta$-tocopherol, $\delta$-tocopherol, and $\delta$-tocotrienol, were not detected in the methanol extracts of either rice bran.

\section{Mutagenicity of rice bran extracts}

Various concentrations of rice bran extracts ranging from 0.2-5.0 mg/plate were used. At the highest concentration of 5 $\mathrm{mg} /$ plate, none of the rice bran extracts showed a killing effect or cytotoxicity to $S$. typhimurium strains TA98 and TA100 in the presence or absence of metabolic activation. In addition, these extracts did not increase the number of revertant colonies by more than 2 times compared to that of spontaneous revertant colonies in both strains, with or without metabolic activation (Table 2). The results indicated that purple and white rice bran extracts had no mutagenicity in $S$. typhimurium strains TA98 and TA100.

\section{Antimutagenicity of rice bran extracts}

Antimutagenic activities of rice bran extracts against standard environmental mutagens in the Salmonella mutation assay using TA98 and TA100 are shown in Figure 3. The non-cytotoxic concentrations of rice bran extracts were used. All rice bran extracts decreased the number of revertant colonies induced by $\mathrm{AFB}_{1}$ and MeIQ in TA98 and TA100, respectively (Fig. 3A and 3B). Moderate inhibition of 2-AF mutagenicity was found in the dichloromethane extracts of rice bran (Fig. 3C). Only the methanol extract of purple rice bran had a mild antimutagenic effect against $\mathrm{B}(\mathrm{a}) \mathrm{P}$ in TA100 (Fig. 3D). These findings suggested that lipophilic and hydrophilic constituents of rice bran extracts affected $\mathrm{AFB}_{1}$ and MeIQ-induced mutagenesis, whereas only lipophilic constituents presented the inhibitory effect on 2-AF-induced mutagenesis. The hydrophilic constituents are proposed as antimutagens against $\mathrm{B}(\mathrm{a}) \mathrm{P}$.

\section{The induction of quinone reductase activity by rice bran extracts}

A marker for screening cancer chemopreventive activity was assayed using murine hepatoma cells (Hepa 1c1c7). The induction of quinone reductase activity in Hepa 1c1c7 tended to increase after treatment with rice bran extracts at the concentration of $200 \mu \mathrm{g} / \mathrm{mL}$. This increase did not reach a 2-fold level of induction as compared to a negative control, although a nearly 4-fold increase of induction was detected after treatment with $\beta$-napthoflavone, a positive control (Table 3).

\section{DPPH radical scavenging activity of rice bran extracts}

A relatively high level of DPPH scavenging activity was observed in the methanol extract of rice brans. The methanol extract of purple rice bran presented the greatest antioxidant capacity (Fig. 4). On the other hand, the dichloromethane extracts of both rice bran had lower antioxidant levels than the methanol extracts. These results were correlated with the amount of phenolic compounds in each rice bran extract.

Table 2. Mutagenicity of rice bran extracts using the Salmonella mutation assay in TA98 and TA100, with or without metabolic activation

\begin{tabular}{|c|c|c|c|c|c|c|c|c|c|}
\hline \multirow{3}{*}{ Treatment } & \multirow{3}{*}{$\begin{array}{c}\text { Concentration } \\
\text { (per plate) }\end{array}$} & \multicolumn{8}{|c|}{ Number of $\mathrm{His}^{+}$revertant colonies } \\
\hline & & \multicolumn{2}{|c|}{ TA98 (+ S9) } & \multicolumn{2}{|c|}{ TA98 $(-$ S9) } & \multicolumn{2}{|c|}{ TA100 (+S9) } & \multicolumn{2}{|c|}{ TA100 (-S9) } \\
\hline & & DCM & $\mathrm{MeOH}$ & DCM & $\mathrm{MeOH}$ & DCM & $\mathrm{MeOH}$ & DCM & $\mathrm{MeOH}$ \\
\hline DMSO & $50 \mu \mathrm{L}$ & \multicolumn{2}{|c|}{$30.1 \pm 2.1$} & \multicolumn{2}{|c|}{$24.7 \pm 0.6$} & \multicolumn{2}{|c|}{$117.7 \pm 5.3$} & \multicolumn{2}{|c|}{$123.8 \pm 4.4$} \\
\hline AF-2 & $0.01 \mu \mathrm{g}$ & \multirow{2}{*}{\multicolumn{2}{|c|}{ - }} & \multicolumn{2}{|c|}{-} & \multicolumn{2}{|c|}{-} & \multicolumn{2}{|c|}{$509.1 \pm 19.4$} \\
\hline AF-2 & $0.1 \mu \mathrm{g}$ & & & \multicolumn{2}{|c|}{$347.0 \pm 16.9$} & \multicolumn{2}{|c|}{-} & \multicolumn{2}{|c|}{-} \\
\hline 2-AA & $0.5 \mathrm{mg}$ & \multicolumn{2}{|c|}{$416.1 \pm 21.1$} & & - & $612.0 \pm$ & $=27.3$ & - & - \\
\hline \multirow{3}{*}{$\begin{array}{l}\text { Purple rice } \\
\text { bran }\end{array}$} & $0.2 \mathrm{mg}$ & $30.7 \pm 2.4$ & $33.0 \pm 3.2$ & $20.1 \pm 1.2$ & $22.6 \pm 0.6$ & $105.4 \pm 3.1$ & $122.9 \pm 3.1$ & $133.1 \pm 6.5$ & $126.4 \pm 3.7$ \\
\hline & $1.0 \mathrm{mg}$ & $28.6 \pm 2.5$ & $35.3 \pm 2.8$ & $18.9 \pm 0.6$ & $22.8 \pm 1.2$ & $107.9 \pm 5.7$ & $116.6 \pm 6.3$ & $139.1 \pm 3.3$ & $126.9 \pm 2.9$ \\
\hline & $5.0 \mathrm{mg}$ & $25.8 \pm 2.5$ & $41.0 \pm 3.1$ & $17.9 \pm 0.5$ & $24.8 \pm 2.3$ & $111.8 \pm 6.7$ & $158.9 \pm 8.0$ & $124.4 \pm 5.2$ & $113.4 \pm 7.6$ \\
\hline \multirow{3}{*}{$\begin{array}{l}\text { White rice } \\
\text { bran }\end{array}$} & $0.2 \mathrm{mg}$ & $32.1 \pm 4.0$ & $31.2 \pm 3.0$ & $19.3 \pm 0.7$ & $23.8 \pm 1.0$ & $104.4 \pm 5.6$ & $117.3 \pm 3.2$ & $112.4 \pm 5.6$ & $119.6 \pm 6.7$ \\
\hline & $1.0 \mathrm{mg}$ & $26.1 \pm 3.5$ & $30.4 \pm 2.2$ & $18.9 \pm 0.5$ & $21.3 \pm 0.9$ & $98.4 \pm 5.4$ & $121.7 \pm 5.6$ & $91.6 \pm 2.9$ & $106.3 \pm 5.0$ \\
\hline & $5.0 \mathrm{mg}$ & $28.8 \pm 3.3$ & $32.6 \pm 2.6$ & $18.1 \pm 0.5$ & $22.2 \pm 0.9$ & $109.4 \pm 6.0$ & $132.7 \pm 4.4$ & $93.7 \pm 3.0$ & $85.0 \pm 1.9$ \\
\hline
\end{tabular}

Values are presented as mean \pm SEM. S9, S9 mix; DCM, dichloromethane extract; MeOH, methanol extract; DMSO, dimethyl sulfoxide; AF2, 2-(2-furyl)-3-(5-nitro-2-furyl) acrylamide; 2-AA, 2-aminoanthracene. 

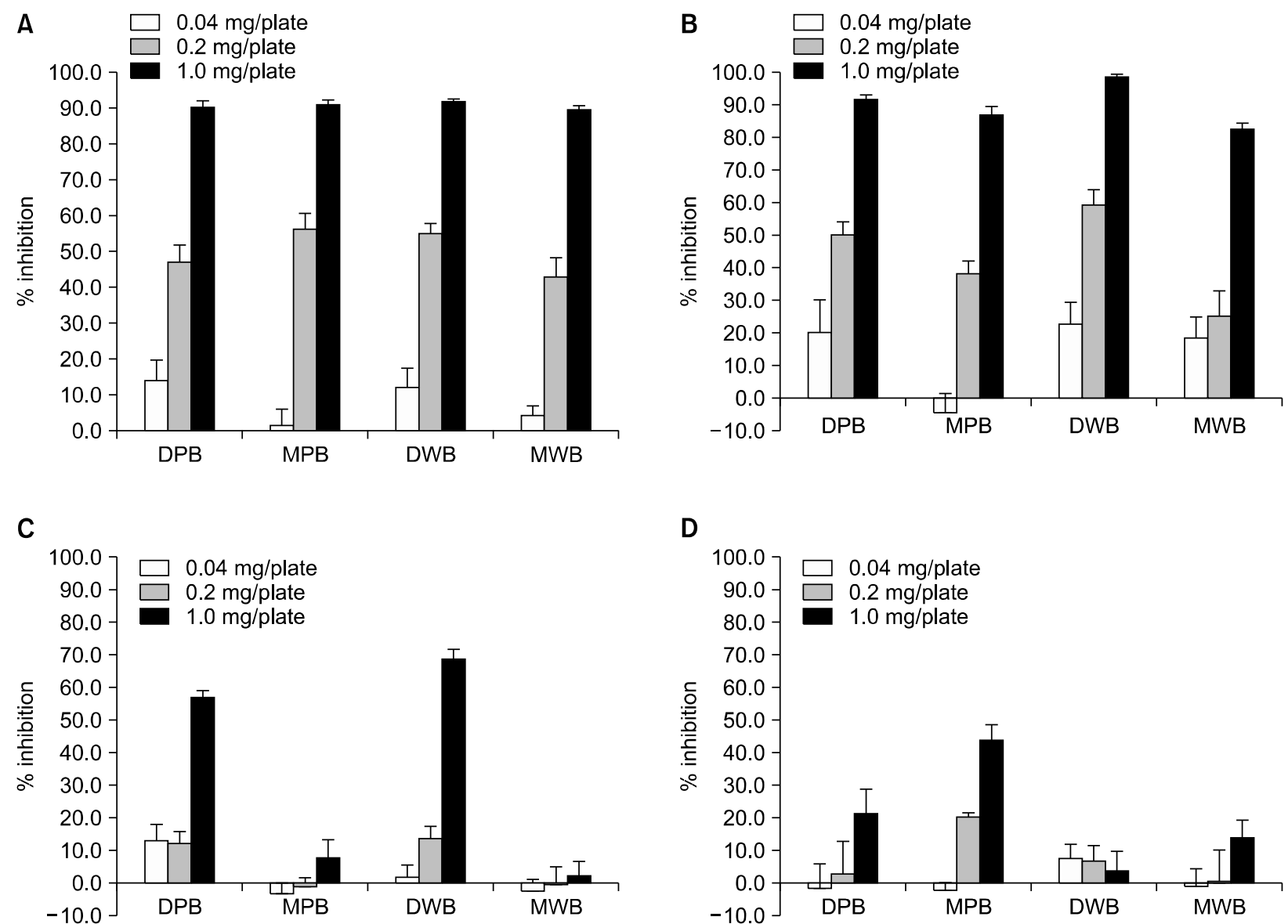

Figure 3. Antimutagenicity of rice bran extracts against standard environmental mutagens. (A) aflatoxin $B_{1}\left(A_{F B}\right)$, (B) 2-amino-3,4 dimethylimidazo [4,5-f] quinoline (MeIQ), (C) 2-aminoflurene (2-AF), and (D) benzo(a)pyrene (B(a)P). DPB, dichloromethane extract of purple rice bran; $\mathrm{MPB}$, methanol extract of purple rice bran; DWB, dichloromethane extract of white rice bran; MWB, methanol extract of white rice bran.

Table 3. Effect of rice bran extracts on the induction of quinone reductase activity in Hepalc1c7 cell lines

\begin{tabular}{|c|c|c|c|c|}
\hline Treatment & Dose (per mL) & $\%$ cell viability & Specific activity & Induction fold \\
\hline $0.4 \%$ dimethylsulfoxide & - & $100.0 \pm 0.0$ & $861 \pm 69$ & $1.00 \pm 0.00$ \\
\hline$\beta$-naphthoflavone & $0.1 \mu \mathrm{g}$ & $109.4 \pm 3.2$ & $3.326 \pm 80$ & $3.88 \pm 0.26$ \\
\hline \multicolumn{5}{|l|}{ Purple rice bran } \\
\hline \multirow[t]{3}{*}{ Dichloromethane } & $50 \mu \mathrm{g}$ & $115.4 \pm 3.8$ & $1,082 \pm 48$ & $1.26 \pm 0.06$ \\
\hline & $100 \mu \mathrm{g}$ & $115.1 \pm 3.6$ & $1,189 \pm 153$ & $1.38 \pm 0.07$ \\
\hline & $200 \mu \mathrm{g}$ & $104.8 \pm 0.9$ & $1,525 \pm 214$ & $1.77 \pm 0.11$ \\
\hline \multirow[t]{3}{*}{ Methanol } & $50 \mu \mathrm{g}$ & $100.2 \pm 1.7$ & $1,033 \pm 53$ & $1.20 \pm 0.04$ \\
\hline & $100 \mu \mathrm{g}$ & $114.2 \pm 1.9$ & $1,084 \pm 10$ & $1.26 \pm 0.09$ \\
\hline & $200 \mu \mathrm{g}$ & $115.0 \pm 4.2$ & $1,351 \pm 35$ & $1.58 \pm 0.14$ \\
\hline \multicolumn{5}{|l|}{ White rice bran } \\
\hline \multirow[t]{3}{*}{ Dichloromethane } & $50 \mu \mathrm{g}$ & $115.4 \pm 3.8$ & $1,131 \pm 82$ & $1.31 \pm 0.05$ \\
\hline & $100 \mu \mathrm{g}$ & $114.6 \pm 2.5$ & $1,310 \pm 129$ & $1.52 \pm 0.03$ \\
\hline & $200 \mu \mathrm{g}$ & $105.0 \pm 1.6$ & $1,627 \pm 166$ & $1.89 \pm 0.07$ \\
\hline \multirow[t]{3}{*}{ Methanol } & $50 \mu \mathrm{g}$ & $101.2 \pm 0.9$ & $1,148 \pm 100$ & $1.33 \pm 0.03$ \\
\hline & $100 \mu \mathrm{g}$ & $114.5 \pm 4.9$ & $1,175 \pm 69$ & $1.37 \pm 0.04$ \\
\hline & $200 \mu \mathrm{g}$ & $114.0 \pm 5.5$ & $1,368 \pm 80$ & $1.59 \pm 0.12$ \\
\hline
\end{tabular}

Values are presented as mean $\pm \mathrm{SD}$. 

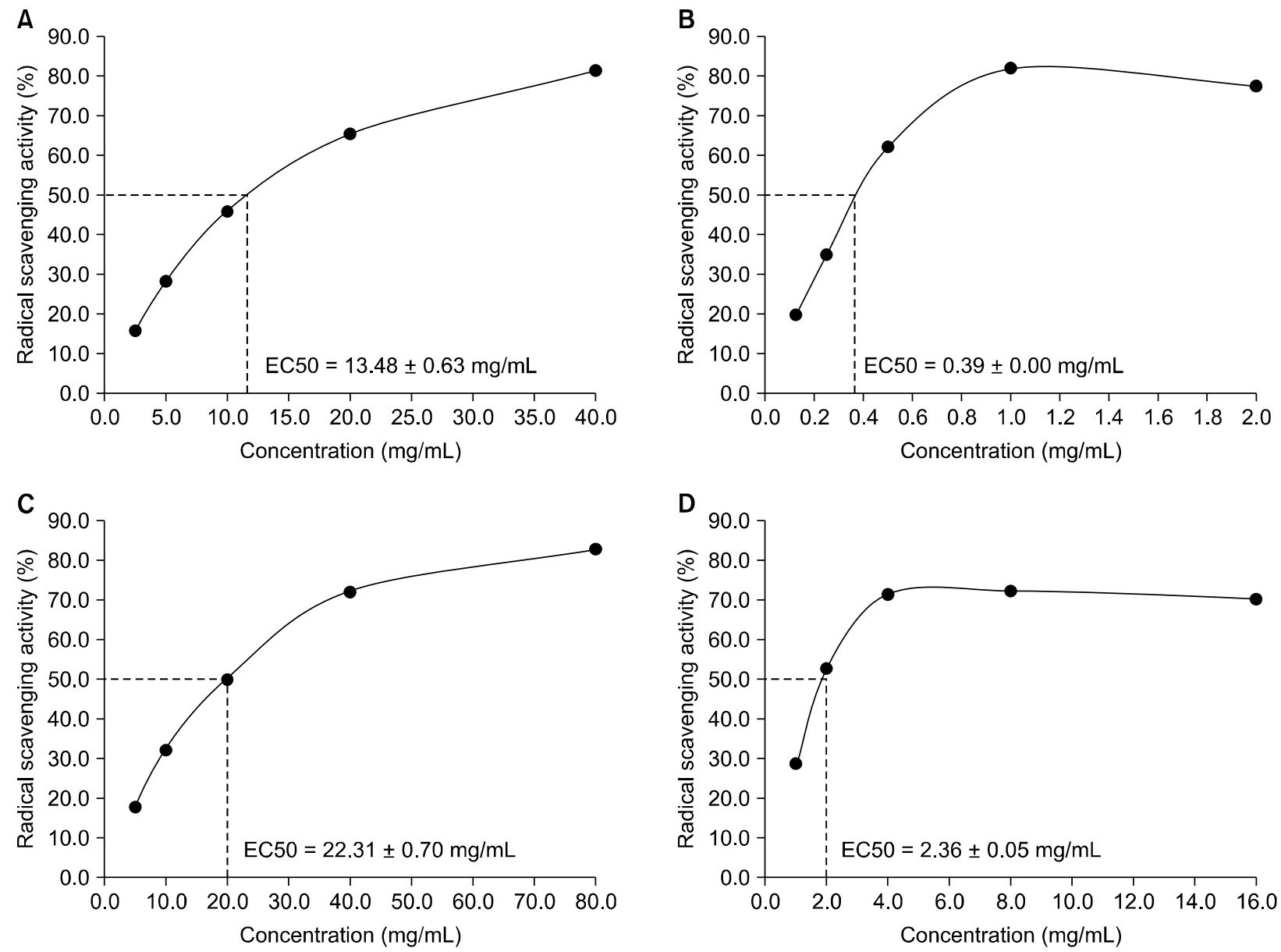

Figure 4. The 2,2-Diphenyl-1-picrylhydrazyl radical scavenging activity of rice bran extracts. (A) Dichloromethane extract of purple rice bran. (B) Methanol extract of purple rice bran. (C) Dichloromethane extract of white rice bran. (D) Methanol extract of white rice bran.

\section{DISCUSSION}

The rice bran extracts examined in the present study had no mutagenic effect on $S$. typhimurium strains TA98 and TA100 in both the presence or absence of metabolic activation. Both purple and white rice bran extracts presented antimutagenic activity against several indirectly acting mutagens. Purple rice bran extracts, particularly the methanol extract, exhibited the greater antimutagenic and antioxidant activities as compared to white rice bran extracts.

DNA mutation is a significant process in chemical carcinogenesis. Mutagens and carcinogens directly react with DNA bases or require xenobiotic metabolizing enzymes to activate their parent forms to highly reactive electrophiles. The binding of ultimate forms with nucleic acids can cause DNA adducts and mutations. ${ }^{21}$ Rice bran presented strong antimutagenicity against $\mathrm{AFB} 1$ and MeIQ in the Salmonella mutation assay. $\mathrm{AFB}_{1}$ and MeIQ are mainly metabolized by cytochrome P450 (CYP450) 1A2 to be ultimate characters, leading to mutagenesis. Both hydrophilic compounds, including phenolic acids, flavonoids, anthocyanins, and phytic acid, and lipophilic compounds, including $\gamma$-oryzanol and vitamin $\mathrm{E}$, were considered as antimutagens in rice bran. They may inhibit CYP1A2 activity, resulting in reduction of revertant colony numbers caused by $\mathrm{AFB}_{1}$ or MeIQ induced mutagenesis. 2-AF is also primarily $\mathrm{N}$-hydroxylated by hepatic CYP1A2 to a reactive $\mathrm{N}$-hydroxy-2-acetylaminofluorene, which can react with guanine residues in nucleic acids. ${ }^{22}$ We found that only the dichloromethane extract of rice bran could inhibit 2-AF induced mutagenesis. 2-AF exhibited greater mutagenicity than $\mathrm{AFB}_{1}$ and MeIQ in this study due to higher numbers of revertant colonies per plate. Thus, the lipophilic parts of rice bran might be stronger CYP1A2 inhibitors than the hydrophilic constituents. However, we found that only the methanol extract of purple rice bran could inhibit $\mathrm{B}(\mathrm{a}) \mathrm{P}$ induced mutagenesis. CYP1A1 is a major phase I metabolizing enzyme that is responsible for activating $\mathrm{B}(\mathrm{a}) \mathrm{P}$ to be a reactive $\mathrm{B}(\mathrm{a}) \mathrm{P}-7,8$-dihydrodiol-9,10-epoxide. ${ }^{23}$ 
Numerous natural polyphenols have been shown to modulate the CYP450 enzyme system, including CYP1A1, 1A2, 2E1, and 3A4. ${ }^{24}$ Dietary flavonoid 5,7-dimethoxyflavone down-regulated B(a)P-induced CYP1A1 expression and activity in HepG2 cells. ${ }^{25}$ Cyanidin-3-glucoside and procyanidins suppressed $\mathrm{B}(\mathrm{a}) \mathrm{P}$ induced CYP1A1 expression in HepG2 cells and ICR mice by inhibiting the transformation of aryl hydrocarbon receptors. ${ }^{26}$ Additionally, ellagic acid decreased CYP1A1 activity in 2,3,7,8-tetrachlorodibenzo-p-dioxin-induced oxidative stress in rats. ${ }^{27}$ These results indicate that antimutagenic agents in Thai rice can inhibit the metabolic activation of several promutagens, resulting in suppression of DNA mutation in a genetic engineered-bacterial model.

Quinones, the intermediates generated from exogenous and endogenous compounds, exert various toxic effects via production of reactive semiquinone by cytochrome $\mathrm{P} 450$ reductase. $^{28}$ NADPH-quinone reductase can detoxify quinones to hydroquinone forms, protecting cells against redox cycling and oxidative stress. ${ }^{28}$ It has been reported that a 2,3-double bond in the $\mathrm{C}$ ring of flavonoids plays a critical role in the induction of quinone reductase. Flavonols, including kaempferol, galangin, quercetin, and myricetin, were the most effective inducers of quinone reductase activity in Hepalclc7 cells, whereas flavanols and flavanone were ineffective. ${ }^{29}$ However, neither purple nor white rice bran extracts induced quinone reductase activity in Hepa 1c1c7 cells. The limited amount of these flavonols in the extracts may be associated with the ineffective quinone reductase induction observed in this study.

Oxidative stress is a process resulting from an imbalance of overproduction of free radicals and limitation of antioxidant systems. ${ }^{30}$ It contributes to numerous pathological conditions, such as obesity, diabetes mellitus, fatty liver disease, and cancer. ${ }^{31}$ Living organisms have integrated antioxidant systems, including enzymatic and non-enzymatic antioxidants, to protect from harmful effects of free radicals. ${ }^{32}$ Many carcinogens may act through the generation of reactive oxygen species, which can react with DNA and cause DNA damage. ${ }^{21}$ Recent investigations have suggested that phytochemicals in pigmented rice bran exhibit potent antioxidative effect. ${ }^{6}$ Similarly, we found that the antioxidant activity of methanol rice bran extract, especially purple rice bran methanol extract, was greater than that of dichloromethane rice bran extracts. This might be due to high amounts of hydrophilic constituents, such as phenolic acids, flavonoids, anthocyanins, and phytic acid. However, low antioxidant activity in dichloromethane extracts may result from the specific reaction of DPPH radical generating system with hydrophilic compounds. The appropriate system for lipophilic compounds should be required for further investigation. Nevertheless, antioxidant activity of several compounds, including phenolic acids, tocopherols, tocotrienols, and $\gamma$-oryzanol, has been identified in rice bran. Among of these compounds, phenolic compounds have demonstrated higher in vitro antioxidant activity than other antioxidants, such as ascorbic acid, tocopherol, and $\gamma$-oryzanol. ${ }^{10}$

In conclusion, the methanol extract of purple rice bran exhibited effective antimutagenic and antioxidant activities. Hydrophilic compounds, including phenolics, and lipophilic compounds, such as vitamin $\mathrm{E}$ and $\gamma$-oryzanol, were considered as potential cancer chemopreventive agents in rice bran. Their inhibitory mechanisms may be due to either blocking mutagenic metabolizing enzymes or scavenging free radicals, which would prevent both initiation and promotion stages of carcinogenesis. These findings support the value of Thai rice as a natural alternative for cancer prevention.

\section{ACKNOWLEDGMENTS}

This work was supported by the grants from the National Research Council of Thailand (NRCT 2015) and the Endowment Fund for Medicine Research, Faculty of Medicine, Chiang Mai University, Thailand (2016).

\section{CONFLICTS OF INTEREST}

No potential conflicts of interest were disclosed.

\section{REFERENCES}

1. Oliveira PA, Colaço A, Chaves R, Guedes-Pinto H, De-La-Cruz PLF, Lopes C. Chemical carcinogenesis. An Acad Bras Cienc 2007; 79:593-616.

2. Ko EY, Moon A. Natural products for chemoprevention of breast cancer. J Cancer Prev 2015;20:223-31.

3. Surh YJ. Cancer chemoprevention with dietary phytochemicals. Nat Rev Cancer 2003;3:768-80.

4. Hu C, Zawistowski J, Ling W, Kitts DD. Black rice (Oryza sativa L. indica) pigmented fraction suppresses both reactive oxygen species and nitric oxide in chemical and biological model systems. J Agric Food Chem 2003;51:5271-7.

5. Moongngarma A, Daomukdaa N, Khumpika S. Chemical compositions, phytochemicals, and antioxidant capacity of rice bran, rice bran layer, and rice germ. APCBEE Procedia 2012;2:73-9.

6. Nam SH, Choi SP, Kang MY, Kozukue N, Friedman M. Antioxidative, antimutagenic, and anticarcinogenic activities of rice bran extracts in chemical and cell assays. J Agric Food Chem 2005:53:816-22 
7. Park MY, Kim JM, Kim JS, Choung MG, Sung MK. Chemopreventive action of anthocyanin-rich black soybean fraction in APC (Min/+) intestinal polyposis model. J Cancer Prev 2015;20:193-201.

8. Yoshida H, Tomiyama Y, Mizushina Y. Lipid components, fatty acids and triacylglycerol molecular species of black and red rices. Food Chem 2010;123:210-5.

9. Suwannakul N, Punvittayagul C, Jarukamjorn K, Wongpoomchai $R$. Purple rice bran extract attenuates the aflatoxin B1-induced initiation stage of hepatocarcinogenesis by alteration of xenobiotic metabolizing enzymes. Asian Pac J Cancer Prev 2015;16:3371-6.

10. Goufo P. Trindade H. Rice antioxidants: phenolic acids, flavonoids, anthocyanins, proanthocyanidins, tocopherols, tocotrienols, g-oryzanol, and phytic acid. Food Sci Nutr 2014:2:75-104.

11. Inboot W, Taya S, Chailungka A, Meepowpan P, Wongpoomchai R. Genotoxicity and antigenotoxicity of the methanol extract of Cleistocalyx nervosum var. paniala seed using a Salmonella mutation assay and rat liver micronucleus tests. Mol Cell Toxicol 2012;8:19-24.

12. Gao Y, Shang C, Saghai Maroof MA, Biyashev RM, Grabau EA, Kwanyuen $\mathrm{P}$, et al. A modified colorimetric method for phytic acid analysis in soybean. Crop Sci 2007;47:1797-803.

13. Punvittayagul C, Sringarm K, Chaiyasut C, Wongpoomchai R. Mutagenicity and antimutagenicity of hydrophilic and lipophilic extracts of Thai northern purple rice. Asian Pac J Cancer Prev 2014:15:9517-22.

14. Engida AM, Kasim NS, Tsigie YA, Ismadji S, Huynh LH, Ju YH. Extraction, identification and quantitative HPLC analysis of flavonoids from sarang semut (Myrmecodia pendan). Ind Crop Prod 2013;41;392-6.

15. Ryu SN, Park SZ, Ho CT. High performance liquid chromatographic determination of anthocyanin pigments in some varieties of black rice. J Food Drug Anal 1998;6:729-36.

16. Azrina A, Maznah I, Azizah AH. Extraction and determination of oryzanol in rice bran of mixed herbarium UKMB; AZ 6807: MR 185, AZ 6808: MR 211, AZ6809: MR 29. ASEAN Food J 2008; 15:89-96.

17. Huang SH, Ng LT. Quantification of tocopherols, tocotrienols, and g-oryzanol contents and their distribution in some commercial rice varieties in Taiwan. J Agric Food Chem 2011;59:11150-9.

18. Maron DM, Ames BN. Revised methods for the Salmonella mutagenicity test. Mutat Res 1983:113:173-215.

19. Prochaska HJ, Santamaria AB, Talalay P. Rapid detection of inducers of enzymes that protect against carcinogens. Proc Natl Acad Sci U S A 1992;89:2394-8.
20. Musa KH, Abdullah A, Kuswandi B, Hidayat MA. A novel high throughput method based on the DPPH dry reagent array for determination of antioxidant activity. Food Chem 2013;141:4102-6.

21. Bhattacharya S. Natural antimutagens: a review. Res J Med Plant 2011:5:116-26.

22. Kim D, Guengerich FP. Cytochrome P450 activation of arylamines and heterocyclic amines. Annu Rev Pharmacol Toxicol 2005; 45:27-49.

23. Schwarz D, Kisselev P, Cascorbi I, Schunck WH, Roots I. Differential metabolism of benzo[a]pyrene and benzo[a]pyrene-7,8-dihydrodiol by human CYP1A1 variants. Carcinogenesis 2001;22: 453-9.

24. Moon YJ, Wang X, Morris ME. Dietary flavonoids: effects on xenobiotic and carcinogen metabolism. Toxicol In Vitro 2006;20: 187-210.

25. Wen X, Walle UK, Walle T. 5,7-Dimethoxyflavone downregulates CYP1A1 expression and benzo[a]pyrene-induced DNA binding in Hep G2 cells. Carcinogenesis 2005;26:803-9.

26. Zhang T, Jiang S, He C, Kimura Y, Yamashita Y, Ashida H. Black soybean seed coat polyphenols prevent B(a)P-induced DNA damage through modulating drug-metabolizing enzymes in HepG2 cells and ICR mice. Mutat Res 2013;752:34-41.

27. Vijaya Padma V, Kalai Selvi P, Sravani S. Protective effect of ellagic acid against TCDD-induced renal oxidative stress: modulation of CYP1A1 activity and antioxidant defense mechanisms. Mol Biol Rep 2014:41:4223-32.

28. Nioi P, Hayes JD. Contribution of $\mathrm{NAD}(\mathrm{P}) \mathrm{H}$ :quinone oxidoreductase 1 to protection against carcinogenesis, and regulation of its gene by the Nrf2 basic-region leucine zipper and the arylhydrocarbon receptor basic helix-loop-helix transcription factors. Mutat Res 2004:555:149-71.

29. Uda Y, Price KR, Williamson G, Rhodes MJ. Induction of the anticarcinogenic marker enzyme, quinone reductase, in murine hepatoma cells in vitro by flavonoids. Cancer Lett 1997;120:213-6.

30. Rolo AP, Teodoro JS, Palmeira CM. Role of oxidative stress in the pathogenesis of nonalcoholic steatohepatitis. Free Radic Biol Med 2012:52:59-69.

31. Matsuda M, Shimomura I. Increased oxidative stress in obesity: implications for metabolic syndrome, diabetes, hypertension, dyslipidemia, atherosclerosis, and cancer. Obes Res Clin Pract 2013;7:e330-41.

32. Valko M, Leibfritz D, Moncol J, Cronin MT, Mazur M, Telser J. Free radicals and antioxidants in normal physiological functions and human disease. Int J Biochem Cell Biol 2007;39:44-84. 